\title{
Periarticular dextrose prolotherapy instead of intra-articular injection for pain and functional improvement in knee osteoarthritis
}

This article was published in the following Dove Press journal:

Journal of Pain Research

17 May 2017

Number of times this article has been viewed

\author{
Zahra Rezasoltani' \\ Mehrdad Taheri² \\ Morteza Kazempour \\ Mofrad $^{3}$ \\ Seyed Amir Mohajerani ${ }^{2}$ \\ 'Department of Physical Medicine \\ and Rehabilitation, AJA University \\ of Medical Sciences, ${ }^{2}$ Department \\ of Anesthesiology and Pain \\ Medicine, Imam Hossein Hospital, \\ Shahid Beheshti University of \\ Medical Sciences, ${ }^{3}$ Department of \\ Anesthesiology and Pain Medicine, \\ AJA University of Medical Sciences, \\ Tehran, Iran
}

Background: Osteoarthritis (OA) is a degenerative disease that can lead to painful and dysfunctional joints. Prolotherapy involves using injections to produce functional restoration of the soft tissues of the joint. Intra-articular injections are controversial because of the introduction of needles into the articular capsule.

Objectives: To compare the effect of periarticular versus intra-articular prolotherapy on pain and disability in patients with knee OA.

Study design: Randomized double-blind controlled clinical trial.

Setting: Single center, university hospital (Imam Hossein Hospital, Tehran, Iran).

Methods: A total of 104 patients with chronic knee OA were enrolled. In the intra-articular group, $8 \mathrm{~mL}$ of $10 \%$ dextrose and $2 \mathrm{~mL}$ of $2 \%$ lidocaine were injected. Injections were repeated at 1 and 2 weeks after the first injection. In the periarticular group, $5 \mathrm{~mL}$ of $20 \%$ dextrose and $5 \mathrm{~mL}$ of $1 \%$ lidocaine were injected subcutaneously at 4 points in the periarticular area. Pain and disability, as assessed using the Western Ontario and McMaster Universities Osteoarthritis Index (WOMAC), were recorded at each follow-up visit at 1, 2, 3, 4, and 5 months post-injection. Results: The visual analog scale score was significantly lower in the periarticular compared with the intra-articular group at the 2-, 3-, 4-, and 5-month visits but not at 1 month. Morning stiffness and difficulty in rising from sitting were improved in both groups and were not significantly different in the peri- and intra-articular groups. Pain, joint locking, and limitation scores were all improved in both groups. Difficulty in walking on flat surfaces or climbing stairs, and sitting and standing pain, were all improved in both groups from 1 to 5 months after treatment. Limitations: WOMAC scores are subjective and could be a limitation of the study.

Conclusion: Periarticular prolotherapy has comparable effects on pain and disability due to knee OA to intra-articular injections, while avoiding risks of complications.

Keywords: prolotherapy, knee, osteoarthritis, periarticular, acupuncture

\section{Introduction}

Osteoarthritis $(\mathrm{OA})$ is a degenerative disease that manifests mainly with painful joints, articular stiffness and decreased function. ${ }^{1}$ Knee OA is currently a leading cause of disability in adults, ${ }^{2}$ and increasing numbers of adults have disabling OA seriously affecting their quality of life. ${ }^{3}$ Although the exact origins of the pain and disability are not clear, various pain generators in the articular capsule, ligaments, synovium, bone, lateral meniscus, and periarticular ligaments and tendons have been implicated. ${ }^{4}$ Current treatments of knee OA include pharmaceuticals, physical therapy, and intraarticular injection of steroids or hyaluronic acid.

Prolotherapy is a novel alternative to total knee arthroplasty (TKA). ${ }^{5}$ It entails injection of materials into or outside the articular space in order to initiate repair 
and functional restoration of soft tissues in the joint. Intraarticular prolotherapy agents can initiate proliferation and regeneration of damaged cartilage tissue. Dextrose has been useful in prolotherapy of knee joints and adjacent compartments. Other traditional compounds used for prolotherapy of knee OA such as non-steroidal anti-inflammatory drugs (NSAIDs), platelet-rich plasma, and botulinum toxin type A are less effective. ${ }^{6}$ In patients with symptomatic knee OA and anterior cruciate ligament laxity, intermittent dextrose injections resulted in significant improvement in anterior cruciate ligament (ACL) laxity, pain, swelling, and range of motion. ${ }^{7,8}$

Several lines of research support the use of intra-articular knee injection for intervention in arthritis. ${ }^{9}$ However, intraarticular injection is still controversial among many clinicians because of the introduction of needles into the articular capsule and possible side effects. Although recent reports on periarticular injection have shown healing effects on articular joints or adjacent structures, ${ }^{10}$ modifications of the techniques may enhance effects on knee OA. Neurofascial (periarticular) prolotherapy involves injections around periarticular sensory nerves and especially their points of fascial penetration, where they reach the subcutaneous plane.

In this study, we evaluated and compared periarticular versus intra-articular injection effect on alleviating pain and improving disability scores of knee OA.

\section{Objective}

We sought to compare the effect of periarticular versus intraarticular prolotherapy on pain and disability in patients with knee OA.

\section{Methods}

The study was reviewed and approved by the Ethics Committee of the Shahid Beheshti University of Medical Sciences. All procedures performed in this study were in accordance with the ethical standards of the institutional and/or national research committee. Information about the study was given comprehensively both orally and in written form to all patients or their accompanying adult. They gave informed consent in writing prior to inclusion in the study. The clinical trial number for this study is IRCT2015102713364N3.

\section{Patient selection}

In a randomized clinical trial, 104 patients with chronic knee OA were enrolled as subjects in the study. The subjects were divided into two groups based on random numbers assigned by a computer to each patient. Intra-articular dextrose prolotherapy was performed in one group and periarticular dextrose prolotherapy in the other group. The physicians who injected the drug solutions and the ones who evaluated patients in follow-up visits were different and were blind to the subject groupings. All demographic variables including age, sex, and body mass index (BMI) were measured and recorded in specified data sheets.

Inclusion criteria were patients with chronic OA over 50 years of age, grade 2 or higher of OA documented by radiology studies, morning stiffness of $<30$ minutes, and 3 months of no response to conservative therapy. Exclusion criteria were severe underlying disease, coagulopathy, history of rheumatologic disorders, diabetes or history of corticosteroid therapy, prolotherapy or intra-articular injection in the past year, and indication for surgical arthroplasty.

\section{Periarticular injection}

All analgesics were discontinued 48 hours before the procedure and for up to 2 weeks after the procedure. In intraarticular group, $8 \mathrm{~mL}$ of $10 \%$ dextrose and $2 \mathrm{~mL}$ of $2 \%$ lidocaine were injected through an infra-patellar approach by a $23 \mathrm{G}$ needle. Injection was repeated at 1 and 2 weeks after first injection. In the periarticular group, $5 \mathrm{~mL}$ of $1 \%$ lidocaine and $5 \mathrm{~mL}$ of $20 \%$ dextrose were mixed in a syringe and $2.5 \mathrm{cc}$ of the solution was injected subcutaneously at 4 points around the knee (Figure 1A) where the periarticular nerves exit the joint capsule (Figure 1A). Two points were
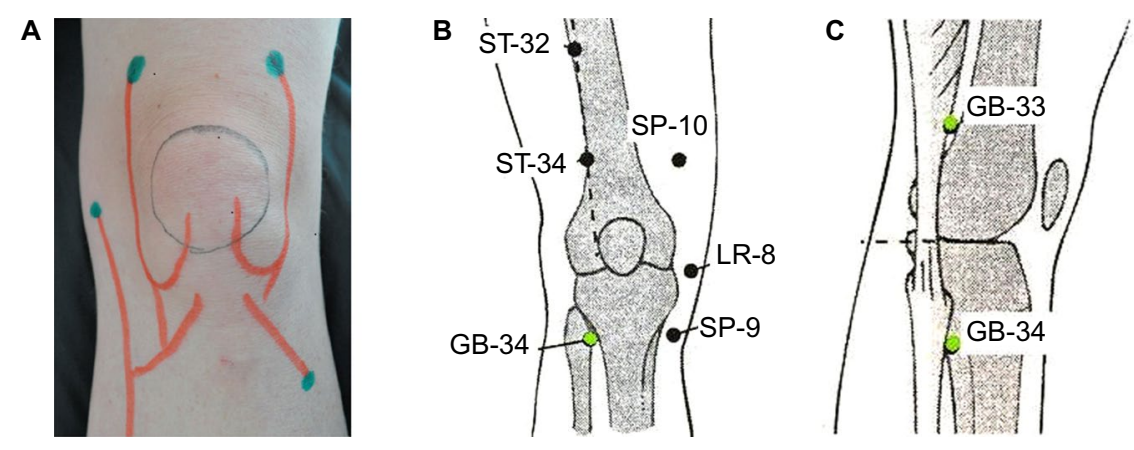

Figure I Points of periarticular injection around the knee (blue dots) and articular nerves of the left knee (red lines).

Notes: (A) Acupuncture points of periarticular injections for knee osteoarthritis in terms of the anteroposterior (B) and lateral (C) view. 
located at upper lateral and medial parts of knee joint, one point at a line medial to knee and one point located at the head of fibula. The injection was performed fan-wise by 2.5 $\mathrm{mL}$ of drug solution $(5 \mathrm{~mL}$ of $1 \%$ lidocaine and $5 \mathrm{~mL}$ of $20 \%$ dextrose) at each point with a $23 \mathrm{G}$ needle. Injections were repeated at 1 and 2 weeks after the first injection. All injections were performed by the same physician.

\section{Pain and disability measurement}

After the injections, patients were scheduled for follow-up visits at 1,2,3,4, and 5 months. At each visit, the visual analog scale (VAS) for pain was measured using a range of $0-100$. The disability index was measured using the Western Ontario and McMaster Universities Osteoarthritis Index (WOMAC) score at each visit, range of motion, and patient satisfaction. In the WOMAC score, the scale of difficulty for different movements is classified based on the following scale: $0=$ none, $1=$ slight, $2=$ moderate, $3=$ very and $4=$ extremely difficult.

\section{Statistical analysis}

Statistical calculations were conducted using SPSS 22 (IBM Corporation, Armonk, NY, USA). The parametric variables were presented as mean $\pm \mathrm{SD}$ and were analyzed by a Student's $t$-test or a Mann-Whitney test; non-parametric variables were analyzed by chi-squared or Fisher's exact test, with $p<0.05$ considered as statistically significant. Sample size was estimated using sample size calculator software with $95 \%$ confidence interval, $p=0.05$ and power of $80 \%$.

\section{Results}

In this study, 110 patients were enrolled, but 5 patients in the periarticular group and 1 patient in the intra-articular group were excluded, and 104 patients remained to the end of study. Age, sex, weight, height, and BMI of patients were not significantly different between two groups (Table 1).

Table I Demographic variables of patients in both groups

\begin{tabular}{llll}
\hline Variables & Periarticular & Intra-articular & $P$-value \\
\hline Patients, $\mathrm{n}$ & 50 & 54 & - \\
Age, years & $63.92 \pm 10.98$ & $63.52 \pm 8.90$ & 0.805 \\
$\begin{array}{l}\text { Gender, } \\
\text { female/male, } \mathrm{n}(\%)\end{array}$ & $38(76.0) / 12(24.0)$ & $40(74.1) / 14(25.9)$ & 0.675 \\
Weight, $\mathrm{kg}$ & $81.52 \pm 14.08$ & $71.88 \pm 50.60$ & 0.002 \\
Height, $\mathrm{cm}$ & $160.86 \pm 7.05$ & $149.08 \pm 44.69$ & 0.605 \\
$\mathrm{BMI}, \mathrm{kg} / \mathrm{m}^{2}$ & $31.57 \pm 5.44$ & $28.92 \pm 3.69$ & 0.006 \\
\hline
\end{tabular}

Note: Data shown as $n$, mean $\pm S D$, or $n(\%)$.

Abbreviation: BMI, body mass index.

\section{Pain score}

The VAS score was significantly lower in the periarticular group compared with the intra-articular group at $2(p=0.001)$, $3(p=0.001), 4(p=0.001)$, and 5 months $(p=0.001)$, but not at 1 month ( $p=0.22$; Figure 2$)$.

\section{WOMAC Score}

Different aspects of the WOMAC score were compared between the two groups using analysis of variance (ANOVA) and Bonferroni tests. Difficulty in rising from sitting was significantly improved in both the periarticular and intraarticular groups at 5 months post-injection $(p<0.05)$. However, the differences between two groups were not significant at any time point (Figure 3). Morning (awakening) stiffness score was significantly improved in both the periarticular and the intra-articular groups beginning at the 1-month visit $(p<0.05)$, but it was not significantly different between peri- and intra-articular groups at any time during the study (Figure 3).

Pain period, joint locking, and joint limitation scores were all improved in both groups in each of the 1-5-month visits; however, joint locking was more improved in the intraarticular group compared with the periarticular group. Pain was significantly improved in the periarticular group from $3.7 \pm 0.8$ at the first visit to $1.8 \pm 0.9$ at the 5 th visit and in the intra-articular group from $3.6 \pm 0.4$ at the first visit to $2.8 \pm 0.5$ at the 5th visit. Pain periods were significantly lower in the periarticular group compared with the intra-articular group at all time points. Joint locking score was significantly higher in the periarticular group than in the intra-articular group at all time points $(p<0.05)$. The joint limitation score was sig-

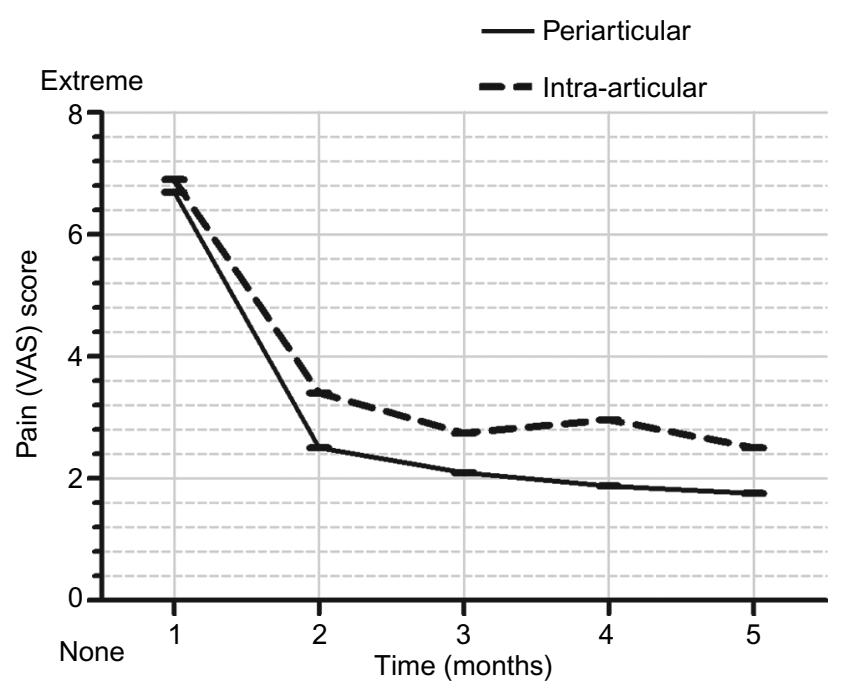

Figure 2 Pain score (VAS) in the periarticular and intra-articular injection groups. Abbreviation: VAS, visual analog scale. 

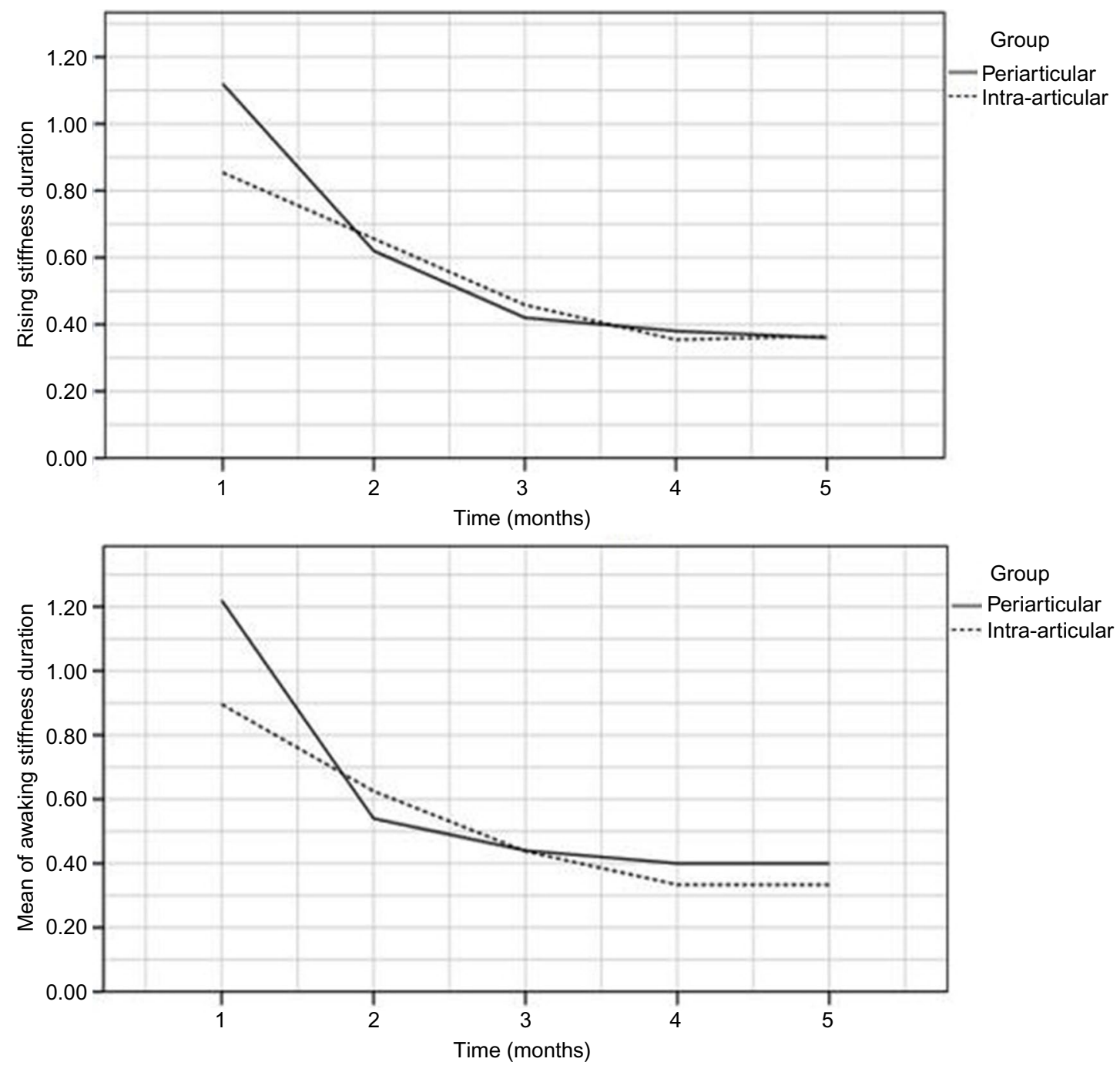

Figure 3 Comparison of rising stiffness duration and awakening stiffness duration in the periarticular and intra-articular injection groups. Notes: $Y$-axes shown in probability, as measured by Mann-Whitney $U$ test.

nificantly lower at all time points in the periarticular group compared with the intra-articular group ( $p<0.05$; Figure 4$)$.

Difficulty in ordinary walking, stair climbing, ordinary activities and underlying pain scores were all improved in both groups from the 1 to the 5 -month visits. Difficulty in walking was not significantly different between the peri- and intra-articular treatment groups at any time point, including the $4(p=0.54)$ and $5(p=0.66)$ month time points. Although difficulty in stair climbing was also not significantly different between peri- and intra-articular groups at any time points $(p>0.05)$, problems with ordinary activities were significantly lower in the periarticular group compared with the intra-articular group at all time points $(p<0.05)$. Mean pain associated with lying down was significantly higher in the periarticular group versus the intra-articular group at all time points $(p<0.05$; Figure 5$)$.

Difficulty in walking on a flat smooth surface, climbing stairs, as well as sitting and standing pain were all improved in both groups during the 1-5-month visits. Difficulty in walking on a flat surface was significantly higher in the periarticular group compared with intra-articular group at all time points $(p<0.05)$. Difficulty in climbing stairs was significantly higher at $2(p=0.008)$ and 3 months $(p=0.011)$ in the periarticular group compared with intra-articular group, but not at $4(p=0.163)$ and $5(p=0.078)$ months. Sitting and standing pain was significantly higher in the periarticular group compared with the intra-articular group at all time points $(p<0.05$; Figure 6). Pain with walking on flat surfaces, climbing stairs, sitting and standing were significantly improved in both groups during all follow-up time points (Figure 6).

\section{Discussion}

In our study, prolotherapy with dextrose periarticular injections around the knee joint showed reductions of pain and disability of knee OA comparable to those associated with intra-articular injections. Both peri- and intra-articular prolo- 

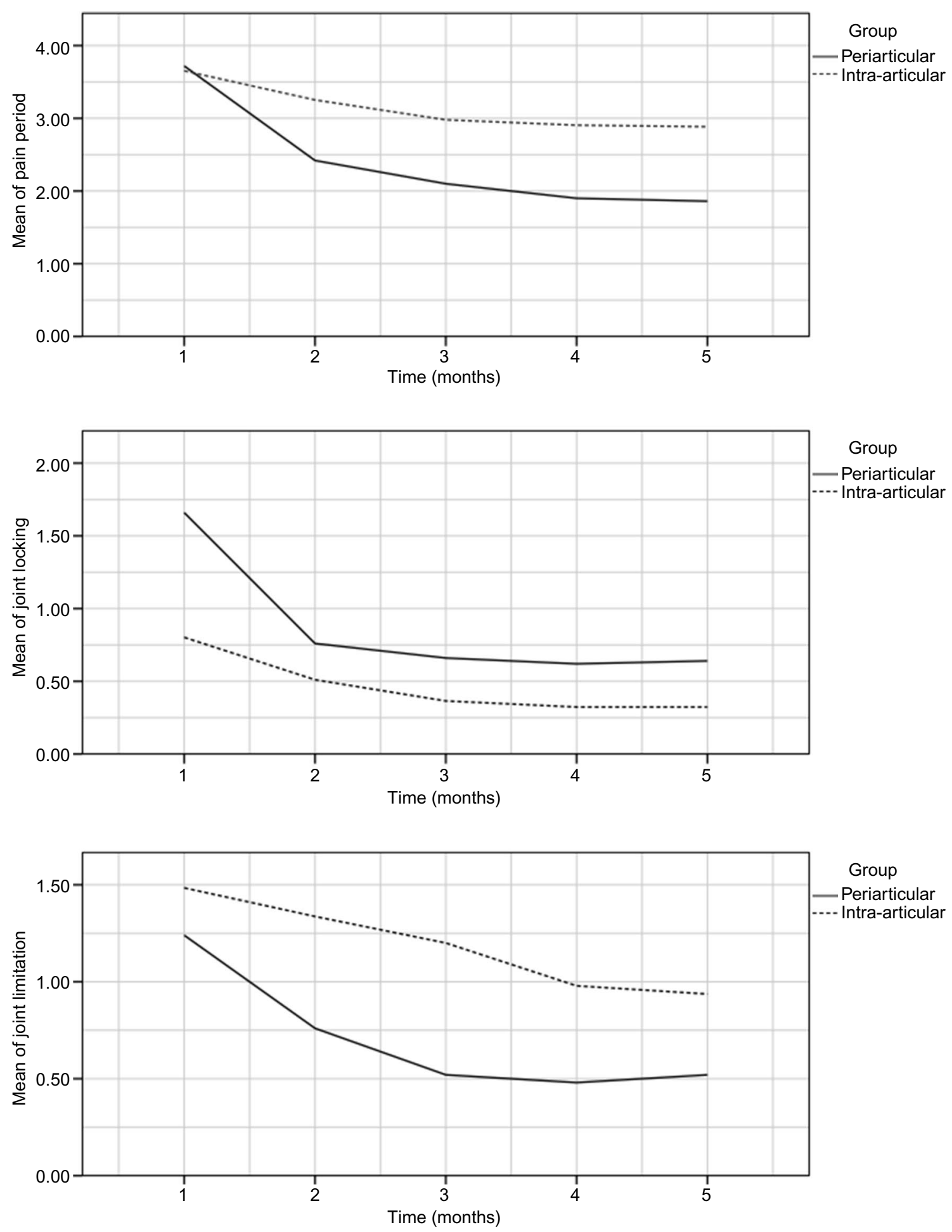

Figure 4 Comparison of the pain period and joint locking and limitation in the periarticular and intra-articular injection groups. Notes: $Y$-axes shown in probability, as measured by Mann-Whitney $U$ test.

therapy patients showed reduced pain and disability of knee OA after 5 months of follow-up. Interestingly, periarticular prolotherapy had better effects on pain scores (VAS) and disability scores (WOMAC) in some respects.

Periarticular prolotherapy showed superior effects on healing of knee disability and WOMAC score compared with intra-articular injections. Pain score was significantly lower at 1-, 2-, 3-, 4-, and 5-month visits in the periarticular group compared with the intra-articular group. Periarticular injections have been suggested in some recent reports for analgesic effect after TKA. ${ }^{11}$ Periarticular injections can significantly reduce the requirements for patient-controlled 

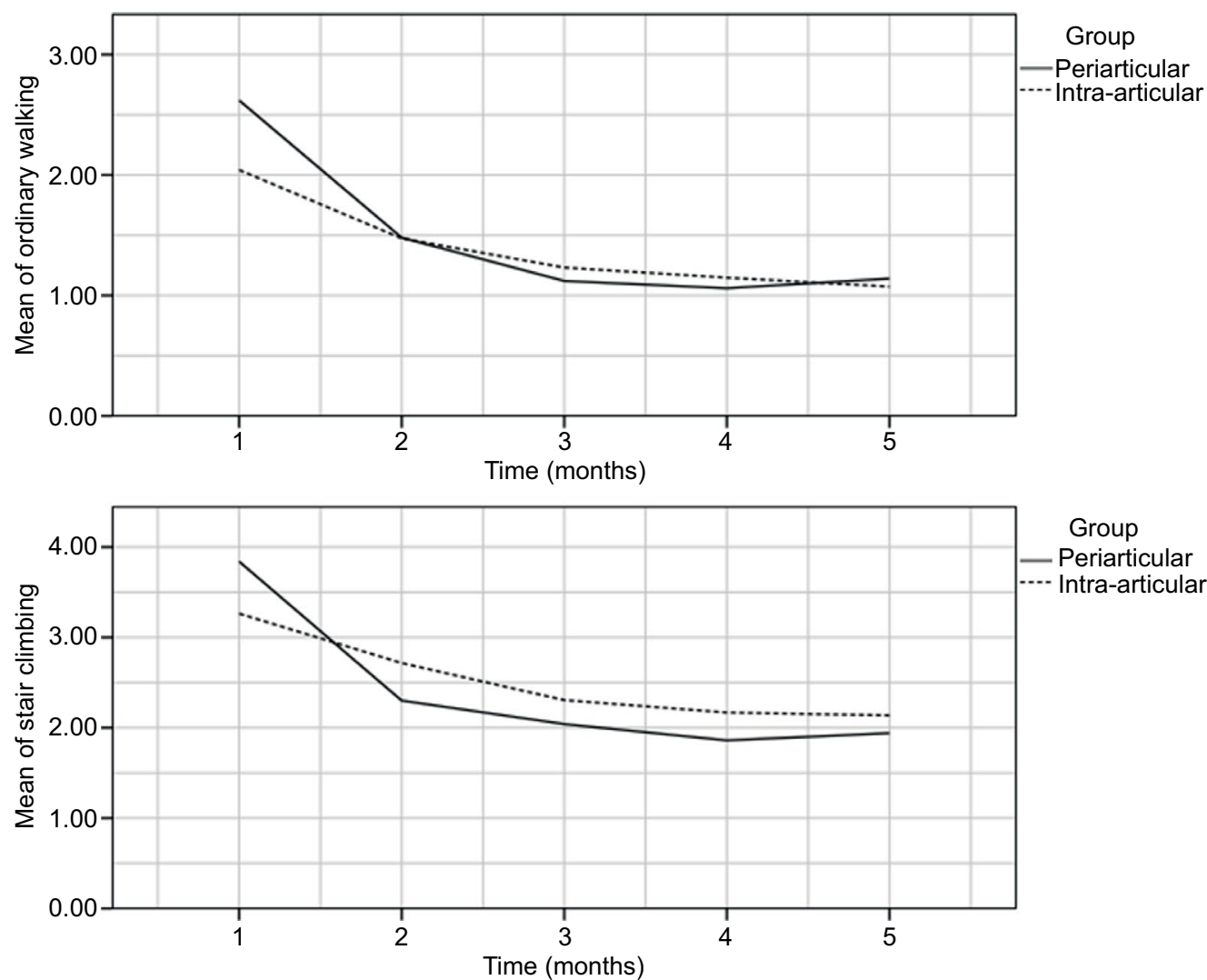

Group

Periarticular

..- Intra-articular

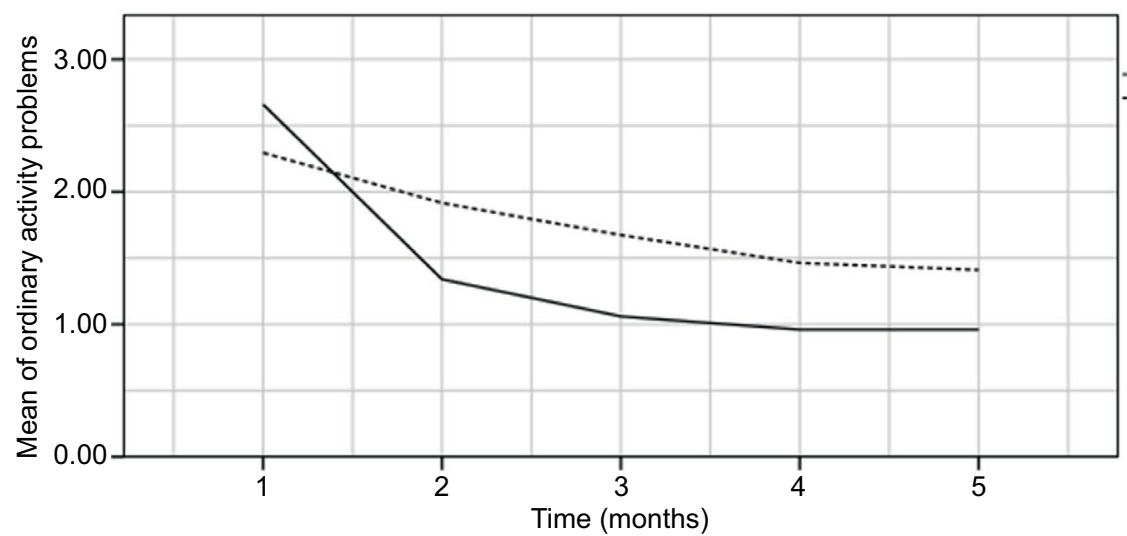

Group

Periarticular

- Intra-articular

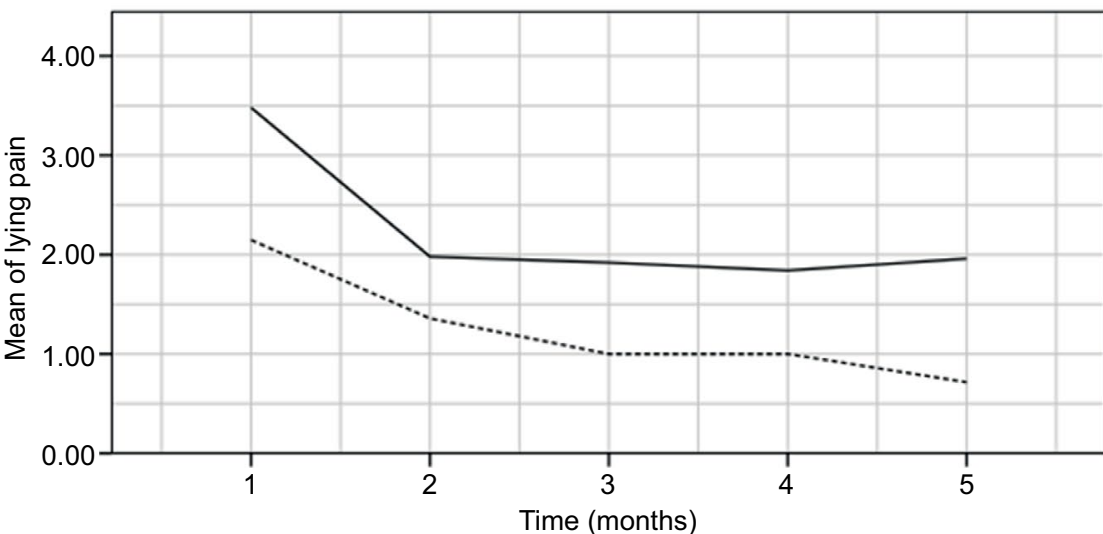

Group

Periarticular - Intra-articular

Figure 5 Comparison of difficulty in walking, stair climbing, ordinary activity problems, and lying pain in the periarticular and intra-articular injection groups. Notes: $Y$-axes shown in probability, as measured by Mann-Whitney $U$ test. 


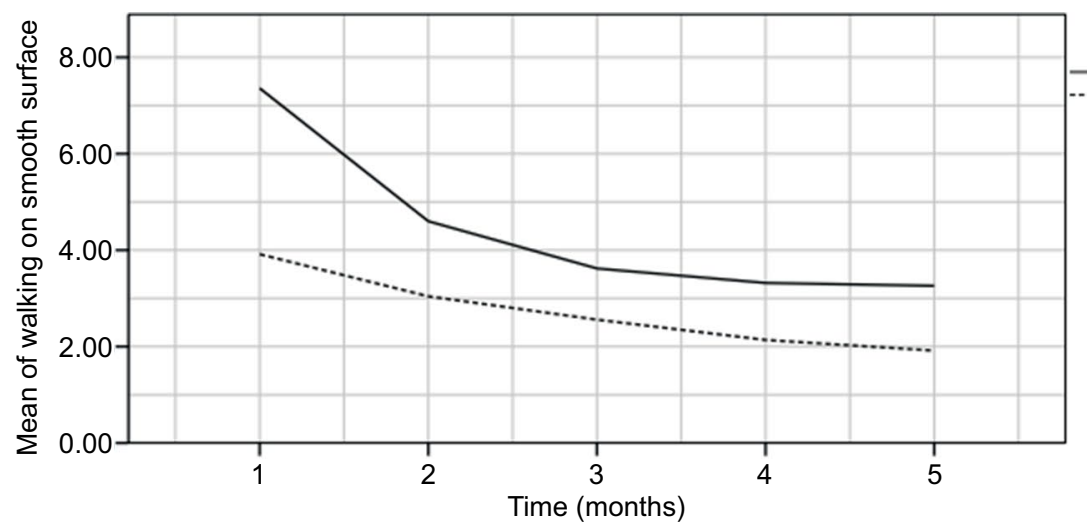

Group

Periarticular

-Intra-articular
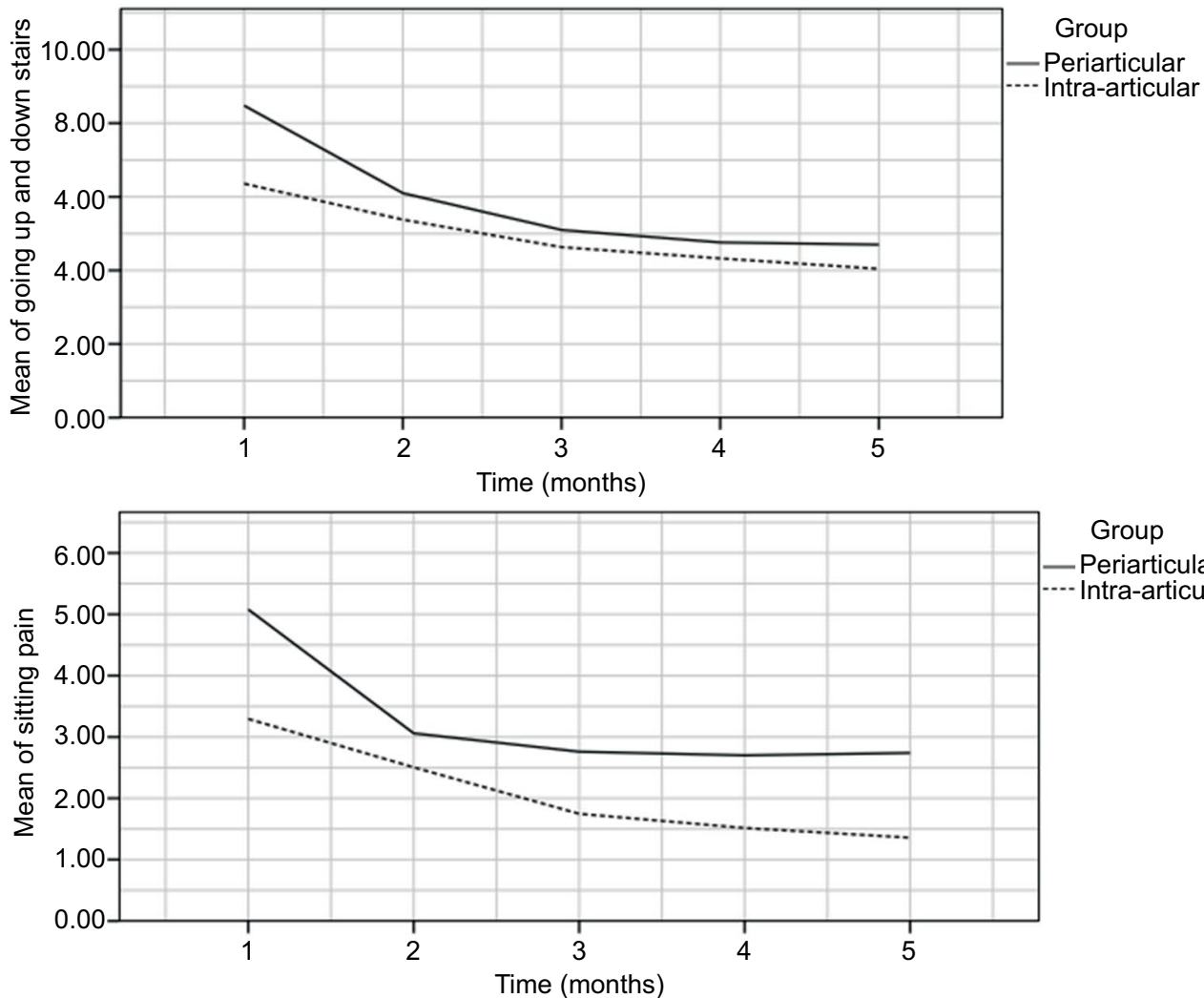

Group

Periarticular - Intra-articular

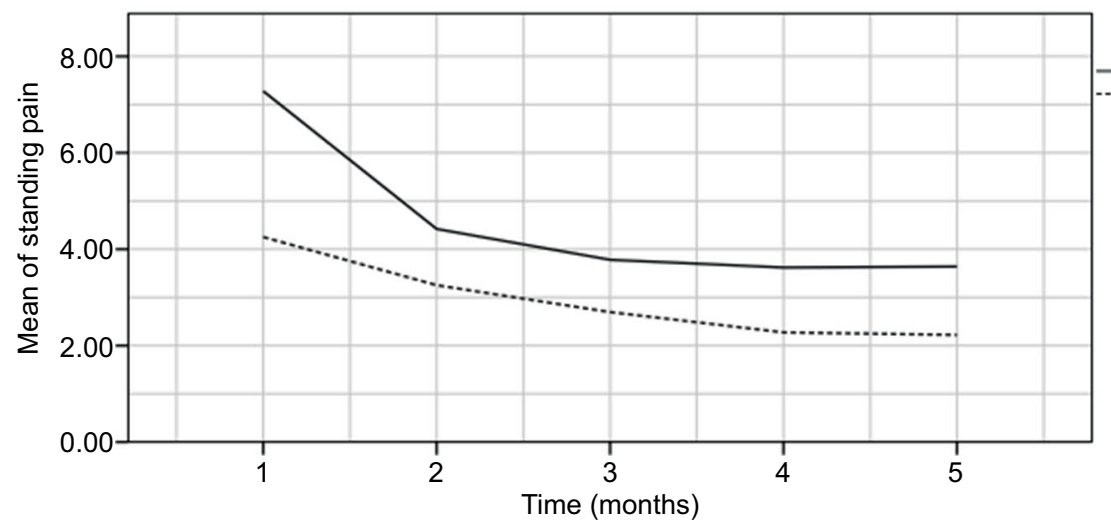

Group

Periarticular Intra-articular

Figure 6 Comparison of walking on smooth surface, going up and down stairs, sitting pain, and standing pain in the periarticular and intra-articular injection groups. Notes: $Y$-axes shown in probability, as measured by Mann-Whitney $U$ test. 
analgesia and can improve patient satisfaction following TKA. ${ }^{12}$ Other than these reports in TKA, in another recent study, periarticular injection showed adjuvant effects to intra-articular prolotherapy. Periarticular injection of lidocaine-corticosteroid improved the clinical efficacy of intra-articular hyaluronic acid alone in patients with knee OA and can be considered a useful adjunctive treatment modality. ${ }^{13}$ On the other hand, intra-articular injections have not been reported in previous studies. Intra-articular injections of hyaluronic acid have only small effects when compared with intra-articular placebo injection. A systematic review has suggested that the intra-articular effects may have been overestimated. $^{14}$

Return of knee function is also an important part of any treatment modality for knee OA. Periarticular prolotherapy in our study showed healing effects in many aspects of knee function that were more marked than with intra-articular prolotherapy. This healing effect was observed particularly in active movements, including walking on flat surfaces, and ascending and descending stairs. The precise molecular targets responsible for the effect of periarticular prolotherapy remain to be elucidated. Periarticular injections around the knee joint ignite the inflammatory reaction in the vicinity of joint capsule. The infiltration of inflammatory cells and cytokines to the periarticular area may enhance blood perfusion in the capsular joint, increase nourishment to the cartilaginous tissue, and enhance regeneration. Hypertonic dextrose has been hypothesized to stimulate healing of chronically injured peri- and intra-articular tissue by increasing inflammatory cytokines. ${ }^{15}$ Other potential mechanisms that have been suggested include stimulating the release of growth factors favoring soft tissue healing and positive neural effects. ${ }^{16,17}$ Volume expansion of local tissue may also produce tissuelevel effects. ${ }^{18}$

Another suggested mechanism for periarticular prolotherapy is neural prolotherapy (NPT), which is based on treatment of neurogenic inflammation and neuronal damage related to the hypothesis of Pybus et al, ${ }^{19}$ who described fiber $\mathrm{C}$ transmission of deep pain signals from knee joint, ligaments, and tendons, as is often seen in OA. Antegrade nerve transmission cause pain perception in the brain, while the reverse nerve impulses travel to the blood vessels where substance $P$ and calcitonin gene-related peptide (CGRP) are released, causing swelling and pain. Two decades later, Lyftogt $^{20}$ used the same concept and treated joint pain and swelling in OA by inhibiting neurogenic inflammation with dextrose prolotherapy. ${ }^{20}$ NPT is given just under the skin close to subcutaneous nerves at weekly intervals. ${ }^{21}$
The sites of injection points were selected based on Hilton's law and chronic constitutional injury (CCI) injection points (Figure 1A) introduced previously. ${ }^{22}$ According to acupuncture sites depicted in Figures $1 \mathrm{~B}$ and $\mathrm{C}$ for knee osteoarthritis, the trigger points are selected for injection of dextrose. These trigger points are accepted in acupuncture as the main points of pain control in knee OA. The notion that these acupuncture sites involve the healing effect in capsular cartilage is hypothetical and this is one of the first studies reporting on use of this method.

Intra-articular injections and puncturing of the joint capsule have been the subject of ongoing debate regarding the management of knee OA because of concerns about side effects and risk of infection. Hemarthrosis and post-injection pain are other possible side effects. The periarticular method avoids capsular injection and its side effects, which gives an additional modality for pain management in knee OA. Limitations of this study included the need to obtain patient consent and also the need for an extended time for followup and patients' adherence to follow-up visits. Both VAS and WOMAC scores are subjective scales and dependent on individual perceptions and therefore are not an objective measurement, which could be a limitation to the study.

\section{Conclusion}

Periarticular prolotherapy significantly improves some aspects of pain and disability of knee OA comparable with intra-articular injections. In addition, periarticular injections avoid risks intrinsic to intra-articular injections. Future studies could determine whether there are synergistic effects of prolotherapy after both intra- and periarticular injections at various time points.

\section{Disclosure}

The authors report no conflicts of interest in this work.

\section{References}

1. Lee J, Song J, Hootman JM, et al. Obesity and other modifiable factors for physical inactivity measured by accelerometer in adults with knee osteoarthritis. Arthritis Care Res (Hoboken). 2013;65(1):53-61.

2. Lawrence RC, Felson DT, Helmick CG, et al; National Arthritis Data Workgroup. Estimates of the prevalence of arthritis and other rheumatic conditions in the United States. Part II. Arthritis Rheum. 2008;58(1):26-35.

3. Yelin E. Cost of musculoskeletal diseases: impact of work disability and functional decline. J Rheumatol Suppl. 2003;68:8-11.

4. Felson DT. The sources of pain in knee osteoarthritis. Curr Opin Rheumatol. 2005;17(5):624-628.

5. Rabago D, Mundt M, Zgierska A, Grettie J. Hypertonic dextrose injection (prolotherapy) for knee osteoarthritis: Long term outcomes. Complement Ther Med. 2015;23(3):388-395.

6. Rabago D, Slattengren A, Zgierska A. Prolotherapy in primary care practice. Prim Care. 2010;37(1):65-80. 
7. Reeves KD, Hassanein KM. Long-term effects of dextrose prolotherapy for anterior cruciate ligament laxity. Altern Ther Health Med. 2003;9(3):58-62.

8. Reeves KD, Hassanein K. Randomized, prospective double-blind placebo-controlled study of dextrose prolotherapy for knee osteoarthritis with or without ACL laxity. Altern Ther Health Med. 2000;6(2):68-74, 77-80.

9. Cheng OT, Souzdalnitski D, Vrooman B, Cheng J. Evidencebased knee injections for the management of arthritis. Pain Med. 2012;13(6):740-753.

10. Kim TW, Park SJ, Lim SH, Seong SC, Lee S, Lee MC. Which analgesic mixture is appropriate for periarticular injection after total knee arthroplasty? Prospective, randomized, double-blind study. Knee Surg Sports Traumatol Arthrosc. 2015;23(3):838-845.

11. Kelley TC, Adams MJ, Mulliken BD, Dalury DF. Efficacy of multimodal perioperative analgesia protocol with periarticular medication injection in total knee arthroplasty: a randomized, double-blinded study. J Arthroplasty. 2013;28(8):1274-1277.

12. Busch CA, Shore BJ, Bhandari R, et al. Efficacy of periarticular multimodal drug injection in total knee arthroplasty. A randomized trial J Bone Joint Surg Am. 2006;88(5):959-963.

13. Rabago D, Zgierska A, Fortney L, et al. Hypertonic dextrose injections (prolotherapy) for knee osteoarthritis: results of a single-arm uncontrolled study with 1-year follow-up. J Altern Complement Med. 2012;18(4):408-414.
14. Lo GH, LaValley M, McAlindon T, Felson DT. Intra-articular hyaluronic acid in treatment of knee osteoarthritis: a meta-analysis. JAMA. 2003;290(23):3115-3121.

15. Jensen KT, Rabago DP, Best TM, Patterson JJ, Vanderby R Jr. Early inflammatory response of knee ligaments to prolotherapy in a rat model. J Orthop Res. 2008;26(6):816-823.

16. Kim SR, Stitik TP, Foye PM, Greenwald BD, Campagnolo DI. Critical review of prolotherapy for osteoarthritis, low back pain, and other musculoskeletal conditions: a physiatric perspective. Am J Phys Med Rehabil. 2004;83(5):379-389.

17. Lyftogt J. Prolotherapy and Achilles tendinopathy: a prospective pilot study of an old treatment. Aust Musculoskelet Med. 2005;10(1):16-19.

18. Robert J de V, Johannes LT, Jan ANV. Platelet-rich plasma for treatment of Achilles tendinopathy. JAMA. 2010;303(17):1696-1697.

19. Pybus PL. Intraneural injections for rheumatoid arthritis and osteoarthritis with control of pain in arthritis in the knee. di Fabio A, editor. Birmingham: Rheumatoid Disease Foundation; 1989:9-22.

20. Lyftogt J. Subcutaneous prolotherapy treatment of refractory knee, shoulder and lateral elbow pain. Aust Musculoskeletal Med. 2007;12(2): $110-112$.

21. Reeves KD. Prolotherapy: Regenerative Injection Therapy. In: Waldman SD, editor. Pain Management. 2nd ed. Philadelphia; Saunders: USA 2011:1-11.

22. Waldman SD, editor. Pain Management. 2nd ed. Philadelphia: Saunders; 2011.

\section{Journal of Pain Research}

\section{Publish your work in this journal}

The Journal of Pain Research is an international, peer reviewed, open access, online journal that welcomes laboratory and clinical findings in the fields of pain research and the prevention and management of pain. Original research, reviews, symposium reports, hypothesis formation and commentaries are all considered for publication.

\section{Dovepress}

The manuscript management system is completely online and includes a very quick and fair peer-review system, which is all easy to use. Visit http://www.dovepress.com/testimonials.php to read real quotes from published authors. 